\title{
Suppressing the endocrine and autonomic stress systems does not impact the emotional stress experience after psychosocial stress
}

\author{
Nida Ali ${ }^{\mathrm{a}, *}$, Jonas P. Nitschke ${ }^{\mathrm{a}}$, Cory Cooperman ${ }^{\mathrm{a}}$, Jens C. Pruessner ${ }^{\mathrm{a}, \mathrm{b}, \mathrm{c}, \mathrm{d}}$ \\ a Department of Psychology, McGill University, 1205 Dr. Penfield Avenue, Montreal, OC, Canada \\ ${ }^{\mathrm{b}}$ McGill Centre for Studies in Aging, Faculty of Medicine, McGill University, Montreal, QC Canada \\ c Douglas Hospital Research Centre, Department of Psychiatry, Faculty of Medicine, McGill University, Montreal, QC Canada \\ d University of Constance, Department of Psychology, Constance, Germany
}

\begin{abstract}
A B S T R A C T
Acute psychosocial stress activates the physiological and endocrine stress systems and increases the subjective emotional experience of stress. While considerable efforts have been made to link changes in the activity of the biological stress systems with changes in the subjective emotional experience of stress, results so far have been mixed, at best. To investigate this association in a study employing experimental manipulation, we pharmacologically suppressed both the autonomic and the endocrine stress responses, and investigated the effects of acute psychosocial stress on the emotional stress experience.

22 healthy men and women received dexamethasone $(2 \mathrm{mg})$ the day before, and propranolol $(80 \mathrm{mg})$ one hour before psychosocial stress induction. A control group $(n=24)$ received placebo pills on each occasion. Salivary cortisol, alpha-amylase and heart-rate responses to stress were assessed before, during and after stress induction. Subjective stress, mood, and state self-esteem assessments were made before and after stress.

In the pharmacological manipulation group, subjects demonstrated no increase in autonomic or endocrine stress response, after exposure to psychosocial stress. Despite these effects, the emotional stress experience was intact in this group and identical to the control group. Participants in the experimental group showed an increase in subjective stress, greater mood dysregulation, and lower state self-esteem following stress exposure, with the response magnitude comparable to the control group. Our findings suggest that at least acutely, the physiological stress arousal systems and the emotional experience of stress are dissociated. This raises important questions about the efficacy of our measurement of subjective stress, and the unique contributions of the autonomic and endocrine responses in the subjective stress experience.
\end{abstract}

\section{Introduction}

In 1884 William James famously asked if one would run away from a wild bear out of fear or whether the fear would come from running away (James, 1884). The ongoing discussion about the interrelationships between the physiological and subjective stress response has some resemblance to this issue - is the experience of stress a result of the activation of the physiological stress systems, or are the systems activated as a result of the experience of stress? Regardless of where one stands on the issue, one prerequisite is the

\footnotetext{
* Corresponding author at: Department of Psychology, McGill University, 1205 Dr. Penfield Avenue, Montreal, Montreal, QC H3A 1B1, Canada. E-mail address: nida.ali@mail.mcgill.ca (N. Ali).
}

consistent association of the physiological and psychological state during stress.

Physiologically, stress related arousal is associated with the activation of the autonomic nervous system (ANS), resulting in elevated heart-rate (HR) and blood pressure, and increased production of downstream stress biomarkers such as salivary alpha-amylase (sAA; (Engert et al., 2011)). In conjunction, the activation of the slower acting hypothalamic-pituitary-adrenal (HPA) axis results in the secretion of corticotropin-releasing-hormone (CRH), adrenocorticotrophic hormone (ACTH), and the subsequent release of cortisol, the most commonly used stress marker in endocrine research (Chrousos and Gold, 1992; Ulrich-Lai and Herman, 2009).

While numerous studies have shown that acute stress activates the physiological stress systems and increases the subjective emotional experience of stress, only a few (e.g., Campbell and Ehlert, 2012; Mauss et al., 2005) have explicitly examined the correspon- 
dence between the physiological and emotional stress responses. While Mauss et al. (2005) speak generally of modest associations between physiological and emotional experiences; Campbell and Ehlert (2012) explicitly review the interrelationships between biological stress markers (such as cortisol and sAA) and psychological stress markers (such as subjective stress and negative mood). Their findings demonstrate that significant correlations exist between the biological and the psychological stress experiences in only about one quarter of all reviewed studies (before even considering the strength of the association). The authors thus named various possible factors for this apparent dissociation, among them differences in assessment approaches and methodologies, as well as mediating factors such as personality traits and appraisal processes.

One method to investigate the interrelationships between the physiological and psychological systems, that has so far been absent from the literature, is to engage in experimental manipulation of the autonomic and/or endocrine aspects of the stress response and observe the subsequent effects on the subjective stress experience. While previous studies have engaged in partial suppression of the stress response by suppressing either the autonomic system or the endocrine system (Andrews et al., 2012; Andrews and Pruessner, 2013; Maheu et al., 2005), none have examined the effects on the subjective psychological stress experience in the complete absence of the autonomic and endocrine stress responses.

Therefore, the goal of the current study was to pharmacologically suppress both, the autonomic and the endocrine stress responses in order to investigate the effects of acute stress exposure purely on psychological stress perception. In contrast to previous paradigms using pharmacological manipulations to alter the functioning of individual stress systems (Andrews et al., 2012; Andrews and Pruessner, 2013; Maheu et al., 2005), we engaged in a double suppression of the ANS and HPA axis, prior to stress exposure. Keeping in line with classical and contemporary theories of emotion (James, 1884; Lange, 1885; Schachter and Singer, 1962), we predicted that the absence of the autonomic and endocrine activation would result in a markedly reduced subjective stress experience. Additional justification for this hypothesis stems from previous studies in our laboratory where we suppressed the ANS using propranolol, and observed a reduction in the subjective stress experience (Andrews and Pruessner, 2013). To the best of our knowledge, no previous study has examined the effect of psychosocial stress on the subjective experience of stress in the absence of autonomic or endocrine response.

\section{Methods and materials}

46 healthy participants (23 men, mean age $=22.43$, $\mathrm{SD}=3.66$, range $=19-32 ; 23$ women, mean age $=22.65, \mathrm{SD}=4.42$, range $=18-35$ ), with no current or history of medical or psychiatric illnesses were recruited for the study. Exclusion criteria included recreational drug use, consuming more than ten alcoholic beverages a week, smoking more than 7 cigarettes a day and using medications that might affect HPA-axis functioning, including oral contraceptives, for women. Women were tested during the luteal phase of their menstrual cycle (17-28 days after the onset of their last menstruation). To establish the luteal phase, women recorded two menstrual cycles prior to testing. Participants provided written informed consent. The study was approved by the McGill University Faculty of Medicine Institutional Review Board.

Participants were randomly assigned to the placebo condition (PLC; $\mathrm{n}=24$ ) or the dexamethasone-propranolol condition (DP; $n=22$ ). To ensure that the investigators and subjects remained blinded to the drug conditions, participants received two pills, one to take at bedtime, the night before testing (placebo or $2 \mathrm{mg}$ of dexamethasone), and one $60 \mathrm{~min}$ before the onset of stress (placebo or $80 \mathrm{mg}$ of propranolol). A physician was on call while the drugs were active to care for participants in case of pharmacological side effects. No adverse events occurred throughout the study.

\subsection{Testing paradigm}

Testing sessions were held at the same time of day on two consecutive days in our laboratory. On day 1, participants arrived between $9 \mathrm{~h} 30$ and $10 \mathrm{~h} 00 \mathrm{a}$ a.m. On arrival they completed the consent form and a questionnaire to confirm the absence of depressive symptomatology (Beck et al., 1996). They then received one pill (placebo or dexamethasone) with instructions to take it at bedtime. A research assistant sent a text message to every participant in the evening, reminding them to take the pill, and confirming the appointment in the morning. Participants were encouraged to respond back with a confirmation that they had taken the pill, although not all subjects did. On the morning of the testing, a research assistant again confirmed that the pill had been taken as instructed.

On day 2, participants received one pill (placebo or propranolol) one hour before the onset of stress. A research assistant was present to administer the pill. Participants were then seated in a waiting room for a 60 -min rest period for the propranolol to take effect. Participants were then exposed to the psychosocial stress paradigm (described below), following which they completed questionnaires to assess stress related changes in state self esteem and mood.

\subsection{Psychosocial stress paradigm}

To induce acute psychosocial stress, subjects were exposed to the Trier Social Stress Test (TSST; Kirschbaum et al., 1993). The TSST consists of a 10-min anticipation phase during which participants are asked to prepare for a job interview. This is followed by a 5-min speech and a 5-min mental arithmetic task, performed in front of trained confederates, one male and one female. The TSST reliably produces significant increases in stress at all levels: cortisol, sAA, HR, and subjective stress (e.g., Ali and Pruessner, 2012; Engert et al., 2011; Kirschbaum et al., 1993; Nater et al., 2005; Rohleder et al., 2004).

\subsection{Stress markers}

Cortisol and sAA were analyzed from saliva samples (Sarstedt Inc., Saint-Léonard, QC, Canada). Cortisol levels (nmol/l) were measured using a time-resolved fluorescence immunoassay described by Dressendorfer et al. (1992). Salivary alpha-amylase (U/ml) levels were determined using the enzyme kinetic method referred to in Engert et al. (2011). HR was measured by an ambulatory sphygmomanometer (A\&D Company, Tokyo, Japan). All measures were anchored to 9 time-points, in 10-min intervals, throughout the experiment from -20 to $+60 \mathrm{~min}$.

\subsection{Psychological assessment}

\subsubsection{Visual Analogue Scale}

The visual analogue scale (VAS) is frequently used to measure the subjective stress experience (Gift, 1989). Using a 10-point VAS, participants responded to the question "How stressed do you feel right now?". Responses ranged from "not at all" to "extremely". The VAS was administered at 9 time-points on testing day 2 , alongside every saliva sample.

\subsubsection{Current Thoughts Scale}

The Current Thoughts Scale (CTS; Heatherton and Polivy, 1991) is a 20 -item scale used to measure transient changes in self-esteem 
along 3 dimensions: performance, social, and appearance. A composite score can also be used to assess state-self esteem as a continuous measure. The CTS was administered twice on day 2 , at baseline prior to stress induction, and immediately after stress. All three subscales and the composite score were used to measure stress-induced changes in state self-esteem.

\subsubsection{Profile of Mood States}

The Profile of Mood States (POMS; Lorr et al., 1971) is a 65item questionnaire that captures temporary emotional states along six dimensions: tension-anxiety, depression-dejection, angerhostility, confusion-bewilderment, vigour-activity and fatigueinertia. A composite measure of total mood disturbance can also be used to assess overall changes in mood. The POMS was administered twice on day 2, at baseline prior to stress induction, and immediately after stress. All six subscales and the composite measure of total mood disturbance were used to assess stress-induced changes in mood.

\subsection{Statistical analyses}

A two-way (drug X sex) MANOVA was conducted with age, BMI, self-esteem, and BDI as dependent variables to ensure that groups did not differ on these variables.

To compare biomarkers of the different stress systems, across groups, the values for each stress biomarker (cortisol, sAA, and HR) were z-standardized across all participants, over all time points. This allowed us to visualize the crosstalk between the different stress systems using identical units for each system. The z-standardized scores were employed for subsequent statistical analyses, and for visualization. Given that sex differences are observed in physiological stress responses, sex was included as a between-subject factor in all analyses.

A repeated measures MANOVA (time $\times \operatorname{drug} \times$ sex) was conducted to examine the effects of drug administration on the physiological stress variables, with the 9 time-points for each ztransformed biomarker: cortisol, sAA and HR as within-subject factors.

To examine the effects of drug administration on the psychological stress responses, repeated measures ANOVAs (time X drug) were conducted for the VAS (all 9 time-points) and composite measures of CTS and POMS (both time points) as within-subject factors.

To assess change in subjective stress over time, area under the curve with respect to increase (AUCi) was computed for the VAS using the trapezoid formula described by Pruessner et al. (2003). To measure the effects of stress on changes in mood and state self-esteem, we computed the delta-peak - absolute change from baseline to peak stress- for the pre and post measures of POMS (all 7 subscales) and the CTS (all 4 subscales).

A two-way MANOVA (drug X sex) was conducted with the AUCi for VAS, and delta peak for each subscale of the POMS and CTS as dependent variables to assess the effects of drug administration on the subjective emotional experience following stress. Greenhouse-Geisser (GG) corrections were performed if assumptions of sphericity were violated. Significant main effects were decomposed using pair-wise comparisons and Bonferroni corrected. Statistical analyses were conducted using SPSS 21 for Macintosh OS X 10.11

\section{Results}

Two participants (women in the PLC condition) had to be excluded from the data analysis because their scores on the BDI exceeded the established limit for mild depression (Beck et al., 1996). Final data analysis was conducted in 44 participants (PLC $\mathrm{n}=22 ; \mathrm{DP} \mathrm{n}=22$ ).
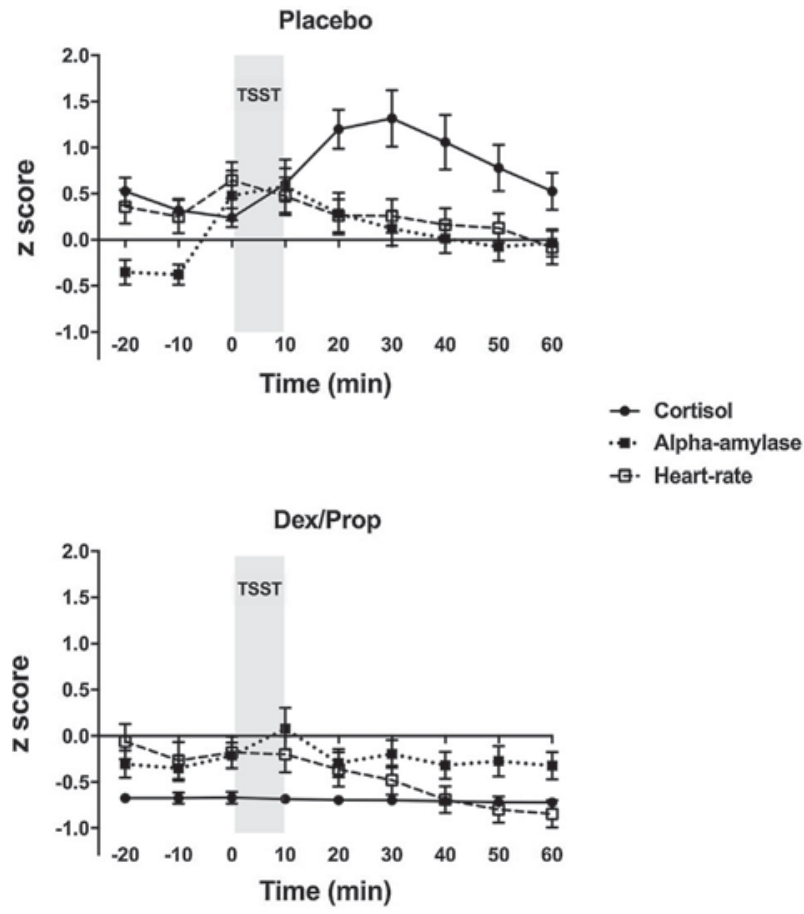

Fig. 1. Z-scores of the change over time in cortisol, alpha-amylase, and heart-rate responses to the TSST, in the Placebo (top) and Dex/Prop (bottom) conditions.

Given that we had a nearly equal distribution of men and women in each drug condition (PLC: 12 men, 10 women; DP: 11 men, 11 women), we conducted a chi-square analysis to examine if there were significant differences between the groups. The results of the Chi-square test (without Yates' continuity correction) revealed that the drug groups did not differ significantly by gender $(\chi \hat{2}(1$, $\mathrm{N}=44)=0.09, \mathrm{p}<0.76, \phi=0.05$, odds ratio $=1.2$ ). These results held when the test was conducted with Yates' continuity correction: $(\chi \hat{2}$ $(1, \mathrm{~N}=44)=0.00, \mathrm{p}=1, \phi=0.05$, the odds ratio is 1.2$)$.

The results of the first set of MANOVAs revealed no significant effects of sex or drug condition on age, self-esteem, BMI or BDI (all $F s<2.44$, ps $>0.1$ ), indicating that the groups were comparable on these factors.

\subsection{Stress biomarkers}

Significant time $\mathrm{X}$ drug interactions were obtained for cortisol, $F(1.59,63.58)=6.29, p=0.006$; $s A A, F(3.06,122.56)=4.37, p=0.006$; and HR, $F(5.12,204.67)=2.41, p=0.037$ (Fig. 1).

Pairwise comparisons revealed that the pharmacological manipulation successfully suppressed all stress biomarkers in the DP condition, i.e. cortisol (PLC: $M=0.71, S E=0.11$; $D P$ : $M=-0.69$, $\mathrm{SE}=0.11$ ), sAA (PLC: $\mathrm{M}=0.10, \mathrm{SE}=0.15$; $\mathrm{DP}: \mathrm{M}=-0.24, \mathrm{SE}=0.15$ ), and HR (PLC: $M=0.28, S E=0.17$; $D P: M=-0.43$, $S E=0.17$ ). Fig. 2 illustrates the stress response between the placebo and DP groups separately, using untransformed values, for cortisol SAA, and HR. There was no significant time $\mathrm{X}$ sex interaction for any stress biomarkers, thus we report the results for both sexes combined.

\subsection{Subjective stress and mood}

The results of the repeated measures ANOVAs (time X drug) revealed a significant main effect of time for each measurement of subjective stress, VAS: $F(3.21,135.17)=29.75, p<0.001$; CTS: $F[1,42]=7.35, p=0.01$; POMS: $F[1,42]=8.99, p=0.005$, indicating a significant change in emotional states after stress. The results fur- 

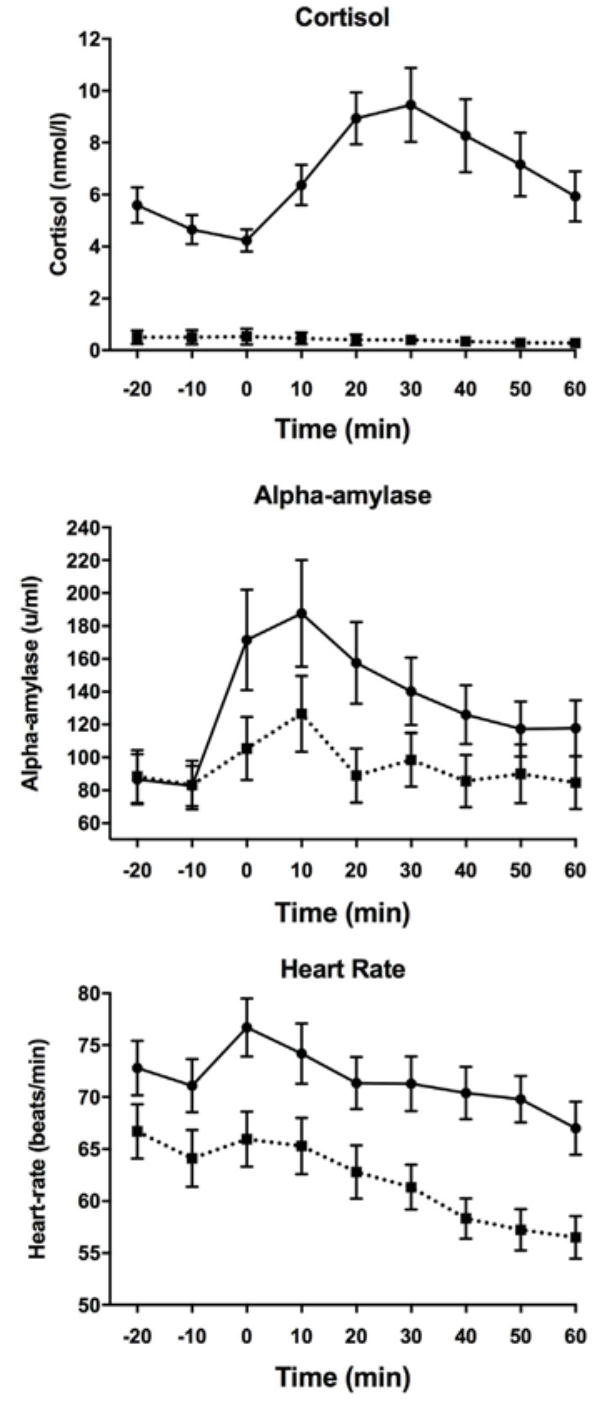

Fig. 2. Changes in stress response over time in the Placebo and Dex/Prop conditions, using untransformed values for cortisol (top), alpha-amylase (middle), and heartrate (bottom).

ther revealed that there was no significant time $\times$ drug interaction, indicating no differences between the PLC and DP conditions.

The results of the MANOVA revealed no significant effects of drug condition for VAS, CTS (all 4 subscales), or POMS (all 7 subscales) (all $F s<2.24, p s>0.14$ ), indicating no significant differences between the PLC and DP groups on the subjective emotional experiences of stress (Figs. 3 and 4). Further, there was no significant drug $X$ sex interaction (all $p s>0.23$ ).

When interpreting a negative result, it is important to take into consideration the statistical power, to observe any effect in the current sample, if there is indeed a difference in the population. We can use the strength of the effect of the pharmacological suppression of the biomarkers to estimate the power, and to find effects on the subjective stress experience. The main effect size $\omega^{2}$ of the drugs on each stress marker was, $\omega^{2}=0.14$ for sAA, $\omega^{2}=0.29$ for HR, and $\omega^{2}=0.75$ for cortisol. Thus, assuming a mean effect size of $\omega^{2}=0.40$ we can estimate a power $T>0.998$ to detect an effect on the subjective stress experience in this sample, if indeed existent in the population, according to the formulas provided by Cohen (1988).

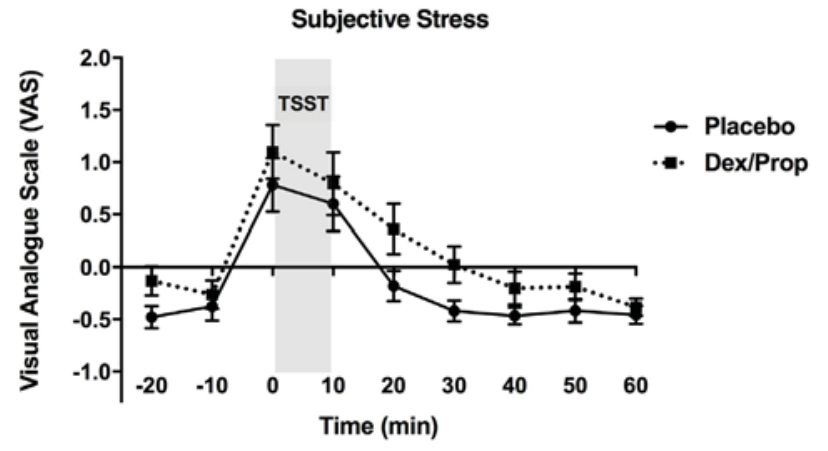

Fig. 3. Z-scores of change over time in subjective stress in response to the TSST, in the Placebo (top) and Dex/Prop (bottom) conditions.
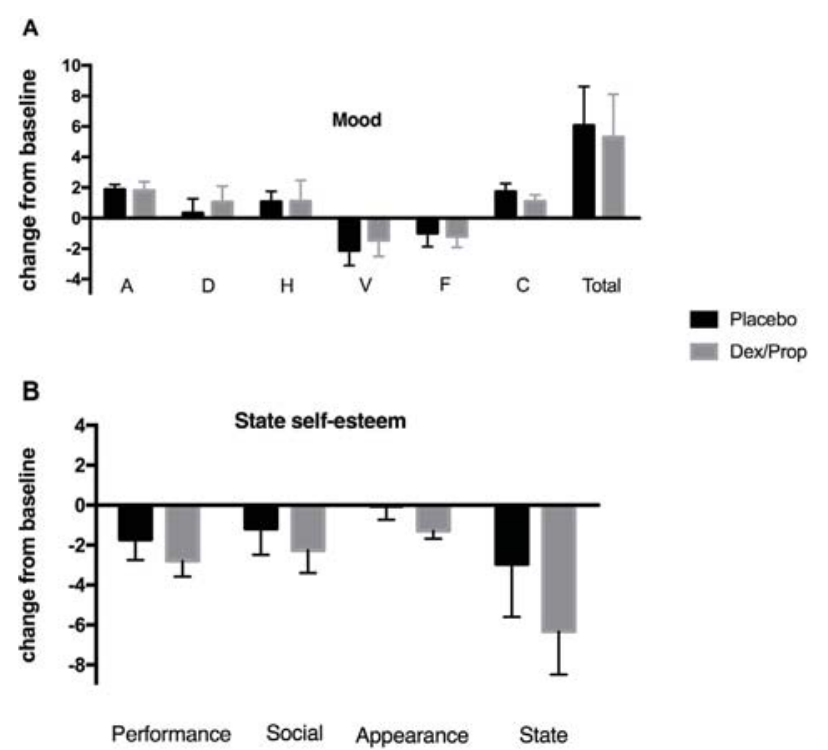

Fig. 4. Effect of TSST on the subjective emotional experiences of stress in the placebo and Dex/Prop groups: A) Mean (+SEM) change from baseline for total and each subscale of the Profile of Mood States (POMS) $(A=$ anxiety, $\mathrm{D}=$ depression, $\mathrm{H}=$ hostility, $\mathrm{V}=$ vigor, $\mathrm{F}=$ fatigue, $\mathrm{C}=$ confusion). $\mathrm{B}$ ) Mean (+SEM) change from baseline for total and each subscale of the Current Thoughts Scale (CTS).

\section{Discussion}

The goal of this study was to pharmacologically inhibit the physiological stress systems (ANS and HPA axis) and investigate the effects of this suppression on the subjective emotional stress experience, in response to acute stress. We predicted that pharmacological blockade would not only impair the reactivity of the HPA axis and ANS in response to acute stress but would also reduce, or dampen, the subjective emotional experience of stress.

Our results indicate that the drug manipulation was effective in impairing the autonomic and endocrine response to psychosocial stress. Specifically, we found that both men and women in the DP condition had significantly blunted cortisol, sAA and HR responses to stress. The respective statistical tests (MANOVAs with time as the repeated measure and the various stress biomarkers as the dependent variables) revealed significant effects of drug condition on the stress response over time (all $p s<0.05$ ), indicating that the usually stressful psychosocial experience did not have a significant effect on the autonomic or endocrine stress systems. To the best of our knowledge, this is the first study that has successfully employed pharmacological suppression of both endocrine and autonomic stress responses in humans. 
In contrast, we found that the subjective emotional stress experience was intact, even in the absence of a significant autonomic or endocrine stress response. Specifically, all participants showed an increase in subjective stress, greater mood dysregulation and lower state self esteem following stress exposure, with no significant differences between the PLC and DP groups. These findings suggest that there is a rather strong dissociation between the physiological stress systems and the subjective emotional experience of stress. The individuals in the DP group reported similar peak amounts of subjective stress as measured via the VAS, as their counterparts in the PLC group. In addition, there was no difference between groups in the subjective stress dynamics over time, with similar baseline, increase from baseline to peak, and return to baseline from peak, measures.

To explain this unexpected finding, several possibilities emerge: First, perhaps our method of suppressing the stress systems was not strong enough, and as a result we can't draw conclusions about the interrelationships between the biological and the psychological stress responses. Several arguments speak against this, both theoretically and as evidenced by our data. For the ANS, propranolol has a long history as a medication used to control hypertension, and has recently received increased attention in the psychological sciences because of its ability to block reconsolidation of emotional memories (e.g., Schwabe et al., 2013, 2012). As a beta-adrenergic receptor blocker, propranolol acts within the limbic system centrally, and on beta-adrenergic receptors peripherally, preventing heart-rate increases during acute stress, and keeping blood pressure in the normal range. Thus, we can be relatively certain that the administration of this drug had the intended effect. This is evidenced by the fact that we observed no significant heart-rate or sAA increases in the experimental group throughout the stress paradigm.

Administering dexamethasone for HPA suppression might not be immediately intuitive, given that it is a synthetic glucocorticoid and leads to an increase in glucocorticoids in the periphery (de Kloet et al., 1974). However, at the level of the pituitary, a dose of more than $1 \mathrm{mg}$ typically results in a complete inhibition of ACTH production for a sustained period of time. Given that dexamethasone does not cross the blood brain barrier in smaller dosages, the negative feedback from the pituitary results in an immediate reduction of cortisol production. Thus, in the periphery, administration of dexamethasone leads to first a hyper-exposure followed by a hypoexposure, while centrally there is an almost immediate reduction of HPA axis activity (Karssen et al., 2005). Since in our study dexamethasone administration took place at bedtime the night before testing, it can be assumed that participants arrived for testing in a hypo-glucocorticoid state, both centrally and in the periphery. This was evidenced by blunted cortisol levels observed in the subjects throughout the study. Thus, we can likely conclude that the suppression of the HPA axis was also effective.

Another possibility could be that the psychological measures to assess the subjective emotional experience of perceived stress are inadequate. While this is not a novel idea (cf. Andrews and Pruessner, 2013; Biondi and Picardi, 1999; Hellhammer and Schubert, 2012), we suggest that the current study with its experimental suppression of the biological stress response reveals this inadequacy more directly. Thus, it could be argued that the measures of psychological stress we have at our disposal are too insensitive to allow any real insight into the psychological state of the individual. This argument can in part be supported by studies showing a blunted physiological or endocrine stress response, in the presence of normal subjective emotional responses to stress (e.g. Childs and de Wit, 2009; Kirschbaum et al., 1999). The current finding could be interpreted as a sign that the general threshold to 'feel stressed' or to acknowledge that a situation is stressful, is probably rather low and that there might be ceiling effects to the subjective experience of stress, which then mask the possible link with physiological or endocrine systems.

If the current measures of psychological stress are indeed inadequate, as a consequence, we would either have to acknowledge this (and stop looking for, or stop reading meaning into, spurious results), or we should aim to develop better measures. While the latter point is beyond the scope of the current manuscript, the authors do wish to direct the reader to some interesting research evolving around implicit measures and biological stress systems, as one alternative (see, for example, the work of Mossink et al., 2015; Wegner et al., 2014; Wegner et al., 2015).

Another possibility is that the measures of subjective emotional experience of stress as employed in this and many other stress studies are not inadequate after all, but simply not associated with the biological stress systems. This too would mean that the assessment of the subjective emotional stress experience in the context of the biological stress response is not as useful as previously thought.

Finally, the question arises about central nervous system processes that might be involved in this dissociation of the physiological and psychological stress response. From our own imaging studies on neural activation during acute stress perception, we know that frontal and temporal lobe structures (orbitofrontal, anterior cingulate, insula cortex, amygdala, hippocampus) seem to be primarily involved in the perception and processing of an acute stressor (Pruessner et al., 2010). Given that these regions are rich in mineralocorticoid (amygdala, hippocampus), glucocorticoid (frontal and temporal lobe, including amygdala and hippocampus), and noradrenergic receptors (amygdala), pharmacological suppression of the autonomic and endocrine stress response systems are likely detected centrally, particularly at the level of the amygdala and hippocampus. However our results indicate that this suppression did not have any effect on the subjective experience of stress, allowing us to hypothesize that these structures might not be immediately and strongly associated with the magnitude of the subjective psychological stress experience.

Taken together, and relating back to the initial question raised by James in 1884, our findings suggest that the emotional experience of stress is not contingent upon perceived cues of physiological arousal. While the results from this study did not reveal any differences between men and women in the subjective experience of stress, our sample size did not allow us to explore sex specific effects, if any. Follow-up studies should consider examining these effects, and assess women in the follicular phase, and on oral contraceptives, given that hormonal and emotional responses are altered in these phases.

Overall, our study demonstrates the efficacy of using pharmacological manipulation to successfully suppress physiological stress arousal, in response to psychosocial stress. The resulting dissociation between the physiological stress response and the emotional experience of stress raises important questions about reliability and validity of our measures of psychological stress, particularly in their relationship with measures of biological stress, and the unique contributions of the autonomic and endocrine responses in the subjective stress experience.

\section{References}

Ali, N., Pruessner, J.C., 2012. The salivary alpha amylase over cortisol ratio as a marker to assess dysregulations of the stress systems. Physiol. Behav. 106, $65-72$

Andrews, J., Pruessner, J.C., 2013. The combined propranolol/TSST paradigm-a new method for psychoneuroendocrinology. PLoS One 8, e57567.

Andrews, J., D'Aguiar, C., Pruessner, J.C., 2012. The combined dexamethasone/TSST paradigm-a new method for psychoneuroendocrinology. PLoS One 7, e38994.

Beck, A.T., Steer, R.A., Ball, R., Ranieri, W., 1996. Comparison of beck depression inventories -IA and -II in psychiatric outpatients. J. Pers. Assess. 67, 588-597. 
Biondi, M., Picardi, A., 1999. Psychological stress and neuroendocrine function in humans: the last two decades of research. Psychother. Psychosom. 68 $114-150$.

Campbell, J., Ehlert, U., 2012. Acute psychosocial stress: does the emotional stress response correspond with physiological responses? Psychoneuroendocrinology 37, 1111-1134.

Childs, E., de Wit, H., 2009. Hormonal, cardiovascular, and subjective responses to acute stress in smokers. Psychopharmacology (Berl.) 203, 1-12.

Chrousos, G.P., Gold, P.W., 1992. The concepts of stress and stress system disorders. Overview of physical and behavioral homeostasis. JAMA 267, 1244-1252.

Cohen, J., 1988. Statistical Power Analysis for the Behavioral Sciences, 2nd ed. L. Erlbaum Associates, Hillsdale, N.J.

de Kloet, E.R., van der Vies, J., de Wied, D., 1974. The site of the suppressive action of dexamethasone on pituitary-adrenal activity. Endocrinology 94, 61-73.

Dressendorfer, R.A., Kirschbaum, C., Rohde, W., Stahl, F., Strasburger, C.J., 1992. Synthesis of a cortisol-biotin conjugate and evaluation as a tracer in an immunoassay for salivary cortisol measurement. J. Steroid Biochem. Mol. Biol. 43, 683-692.

Engert, V., Vogel, S., Efanov, S.I., Duchesne, A., Corbo, V., Ali, N., Pruessner, J.C., 2011 Investigation into the cross-correlation of salivary cortisol and alpha-amylase responses to psychological stress. Psychoneuroendocrinology 36, 1294-1302.

Gift, A.G., 1989. Visual analogue scales: measurement of subjective phenomena. Nurs. Res. 38, 286-288.

Heatherton, T.F., Polivy, J., 1991. Development and validation of a scale for measuring state self-esteem. J. Pers. Soc. Psychol. 60, 895-910.

Hellhammer, J., Schubert, M., 2012. The physiological response to Trier Social Stress Test relates to subjective measures of stress during but not before or after the test. Psychoneuroendocrinology 37, 119-124.

James, W., 1884. II.-What is an emotion? Mind, 188-205.

Karssen, A.M., Meijer, O.C., Berry, A., Sanjuan Pinol, R., de Kloet, E.R., 2005. Low doses of dexamethasone can produce a hypocorticosteroid state in the brain. Endocrinology 146, 5587-5595.

Kirschbaum, C., Pirke, K.M., Hellhammer, D.H., 1993. The 'Trier Social Stress Test'-a tool for investigating psychobiological stress responses in a laboratory setting. Neuropsychobiology 28, 76-81.

Kirschbaum, C., Kudielka, B.M., Gaab, J., Schommer, N.C., Hellhammer, D.H., 1999. Impact of gender, menstrual cycle phase, and oral contraceptives on the activity of the hypothalamus-pituitary-adrenal axis. Psychosom. Med. 61, $154-162$.

Lange, C.G., 1885. Om sindsbevaegelser; et psyko-fysiologisk studie. Lund.

Lorr, M., McNair, D., Droppleman, L., 1971. Manual: Profile of Mood States. Educational and Industrial Testing Service, San Diego, CA
Maheu, F.S., Joober, R., Lupien, S.J., 2005. Declarative memory after stress in humans: differential involvement of the beta-adrenergic and corticosteroid systems. J. Clin. Endocrinol. Metab. 90, 1697-1704.

Mauss, I.B., Levenson, R.W., McCarter, L., Wilhelm, F.H., Gross, J.J., 2005. The tie that binds? Coherence among emotion experience, behavior, and physiology. Emotion 5, 175-190.

Mossink, J.C., Verkuil, B., Burger, A.M., Tollenaar, M.S., Brosschot, J.F., 2015 Ambulatory assessed implicit affect is associated with salivary cortisol. Front. Psychol. 6, 111.

Nater, U.M., Rohleder, N., Gaab, J., Berger, S., Jud, A., Kirschbaum, C., Ehlert, U., 2005. Human salivary alpha-amylase reactivity in a psychosocial stress paradigm. Int. J. Psychophysiol. 55, 333-342.

Pruessner, J.C., Kirschbaum, C., Meinlschmid, G., Hellhammer, D.H., 2003. Two formulas for computation of the area under the curve represent measures of total hormone concentration versus time-dependent change. Psychoneuroendocrinology 28, 916-931.

Pruessner, J.C., Dedovic, K., Pruessner, M., Lord, C., Buss, C., Collins, L., Dagher, A., Lupien, S.J., 2010. Stress regulation in the central nervous system: evidence from structural and functional neuroimaging studies in human populations 2008 Curt Richter Award Winner. Psychoneuroendocrinology 35, 179-191.

Rohleder, N., Nater, U.M., Wolf, J.M., Ehlert, U., Kirschbaum, C., 2004. Psychosocia stress-induced activation of salivary alpha-amylase: an indicator of sympathetic activity? Ann. N. Y. Acad. Sci. 1032, 258-263.

Schachter, S., Singer, J.E., 1962. Cognitive, social, and physiological determinants of emotional state. Psychol. Rev. 69, 379-399.

Schwabe, L., Nader, K., Wolf, O.T., Beaudry, T., Pruessner, J.C., 2012. Neural signature of reconsolidation impairments by propranolol in humans. Biol. Psychiatry 71, 380-386.

Schwabe, L., Nader, K., Pruessner, J.C., 2013. beta-Adrenergic blockade during reactivation reduces the subjective feeling of remembering associated with emotional episodic memories. Biol. Psychol. 92, 227-232.

Ulrich-Lai, Y.M., Herman, J.P., 2009. Neural regulation of endocrine and autonomic stress responses. Nat. Rev. Neurosci. 10, 397-409.

Wegner, M., Schuler, J., Budde, H., 2014. The implicit affiliation motive moderates cortisol responses to acute psychosocial stress in high school students. Psychoneuroendocrinology 48, 162-168.

Wegner, M., Schuler, J., Scheuermann, K.S., Machado, S., Budde, H., 2015. The implicit power motive and adolescents' salivary cortisol responses to acute psychosocial stress and exercise in school. CNS Neurol. Disord. Drug Targets $14,1219-1224$. 